Article

\title{
Underlying Histopathology Determines Response to Oxidative Stress in Cultured Human Primary Proximal Tubular Epithelial Cells
}

\author{
Muhammad Ali Khan ${ }^{1,2,3,4,5,6}$, Xiangju Wang ${ }^{3,4}$, Kurt T.K. Giuliani ${ }^{2,3,4}$, Purba Nag ${ }^{3,4}($, \\ Anca Grivei $^{3,4} \oplus$, Jacobus Ungerer ${ }^{2,3}$, Wendy Hoy ${ }^{1,7}$, Helen Healy ${ }^{1,3,4,7}$, Glenda Gobe ${ }^{1,2,5,7, \dagger}$ \\ and Andrew J. Kassianos $3,4,7, t, *$ (C) \\ 1 NHMRC CKD CRE (CKD.QLD), University of Queensland, Brisbane 4029, Queensland, Australia; \\ Muhammad.A.Khan@uq.edu.au (M.A.K.); W.Hoy@uq.edu.au (W.H.); Helen.Healy@health.qld.gov.au (H.H.); \\ G.Gobe@uq.edu.au (G.G.) \\ 2 Faculty of Medicine, University of Queensland, Brisbane 4006, Queensland, Australia; \\ Kurt.Giuliani@uqconnect.edu.au (K.T.K.G.); Jacobus.Ungerer@health.qld.gov.au (J.U.) \\ 3 Conjoint Internal Medicine Laboratory, Chemical Pathology, Pathology Queensland, Brisbane 4029, \\ Queensland, Australia; Xiangju.Wang@qimrberghofer.edu.au (X.W.); \\ Purba.Nag@qimrberghofer.edu.au (P.N.); Anca.Grivei@qimrberghofer.edu.au (A.G.) \\ 4 Kidney Health Service, Royal Brisbane and Women's Hospital, Brisbane 4029, Queensland, Australia \\ 5 Kidney Disease Research Collaborative, Princess Alexandra Hospital and University of Queensland, \\ Translational Research Institute, Brisbane 4102, Queensland, Australia \\ 6 Department of Pharmacy, Bangabandhu Sheikh Mujibur Rahman Science and Technology University, \\ Gopalganj 8100, Dhaka, Bangladesh \\ 7 Centre for Chronic Disease, Faculty of Medicine, University of Queensland, \\ Brisbane 4029, Queensland, Australia \\ * Correspondence: Andrew.Kassianos@qimrberghofer.edu.au; Tel.: +61-7-3362-0488 \\ + These authors contributed equally to this work.
}

Received: 24 December 2019; Accepted: 13 January 2020; Published: 15 January 2020

check for updates

\begin{abstract}
Proximal tubular epithelial cells (PTEC) are key players in the progression of kidney diseases. PTEC studies to date have primarily used mouse models and transformed human PTEC lines. However, the translatability of these models to human kidney disease has been questioned. In this study, we investigated the phenotypic and functional response of human primary PTEC to oxidative stress, an established driver of kidney disease. Furthermore, we examined the functional contribution of the underlying histopathology of the cortical tissue used to generate our PTEC. We demonstrated that human primary PTEC from both histologically 'normal' and 'diseased' cortical tissue responded to $\mathrm{H}_{2} \mathrm{O}_{2}$-induced oxidative stress with significantly elevated mitochondrial superoxide levels, DNA damage, and significantly decreased proliferation. The functional response of 'normal' PTEC to oxidative stress mirrored the reported pathogenesis of human kidney disease, with significantly attenuated mitochondrial function and increased cell death. In contrast, 'diseased' PTEC were functionally resistant to oxidative stress, with maintenance of mitochondrial function and cell viability. This selective survival of 'diseased' PTEC under oxidizing conditions is reminiscent of the in vivo persistence of maladaptive PTEC following kidney injury. We are now exploring the impact that these differential PTEC responses have in the therapeutic targeting of oxidative stress pathways.
\end{abstract}

Keywords: human primary proximal tubular epithelial cells; oxidative stress; acute kidney injury; chronic kidney disease 


\section{Introduction}

Kidney disease is a major public health problem, affecting approximately $10 \%$ of populations in industrialized countries [1]. Most notably, the global burden of kidney disease continues to rise, driven by population growth, ageing, and the increased prevalence of diabetes and hypertension [2]. The development of disease within the kidney is characterized on renal biopsy by microstructural patterns of pathology, including arteriosclerosis, glomerulosclerosis, and tubular atrophy/interstitial fibrosis [3]. Regardless of the diverse etiologies underlying these histopathological changes, oxidative stress is a common and key pathobiological driver of kidney injury $[4,5]$.

Oxidative stress refers to an elevation in levels of intracellular reactive oxygen species (ROS) that initiate damage to lipids, proteins, and nucleic acids [6]. ROS, including mitochondrial superoxide $\left(\mathrm{O}_{2}{ }^{-}\right)$, are natural byproducts of aerobic metabolism, and have significant functional roles in intracellular signaling and homeostasis. However, an imbalance between the cellular production of ROS and the capacity of cells to neutralize them results in oxidative stress [7]. In turn, oxidative stress drives mitochondrial dysfunction associated with mitochondrial swelling and the loss of mitochondrial membrane potential (MMP), leading to altered gene expression and cell death via apoptotic or necrotic pathways [5].

Oxidative stress disrupts all functional aspects of the kidney, including proximal tubular reabsorption [8]. The epithelial cells of the proximal tubules (PTEC) are a major source of ROS in the kidney due to their high mitochondrial density and the immense energy demands required to support their reabsorption of vital molecules from the glomerular filtrate [4]. As a result, PTEC are particularly susceptible to oxidant-induced damage and death [9]. The maladaptive repair mechanisms of PTEC in response to injury, including oxidative stress, are established as central mediators of kidney disease progression. However, previous studies of oxidative stress in PTEC have focused primarily on mouse models or cell lines, including transformed human PTEC lines [8]. We propose to translate this body of work into human primary PTEC, to examine the pathobiology of oxidant-induced kidney disease in a biological system of physiological relevance to humans. The oxidative stress response of human primary PTEC may, however, be affected by the underlying histopathology of the cortical tissue used to generate these PTEC. This has yet to be investigated.

In this present study, human primary PTEC were isolated from the macroscopically 'healthy' portion of kidney cancer nephrectomies and stratified as normal and diseased PTEC based on the histological absence or presence of a coexisting pathology (glomerulosclerosis, arteriosclerosis, tubulointerstitial fibrosis, or tubular atrophy) within the kidney cortical tissue. We demonstrate that normal PTEC respond to oxidative stress as anticipated, with significantly increased mitochondrial dysfunction and cell necrosis. In contrast, under equivalent conditions of oxidative stress, diseased PTEC maintain mitochondrial function and cell viability. These findings are in line with the in vivo survival and accumulation of maladaptive PTEC that inhibits tubular regeneration and contributes to renal impairment after kidney injury. We propose that our novel results provide a clinically-relevant model by which to unlock the cellular and molecular pathways of oxidant-induced human kidney disease.

\section{Results}

\subsection{Significantly Increased Levels of Oxidative Stress in Histologically 'Diseased' Kidney Cortical Tissue}

Kidney cortical tissue was obtained from the macroscopically-healthy portion of renal cell carcinoma (RCC) nephrectomies from a total of ten donors with preserved kidney function. Specimens were grouped into histologically 'normal' ( $n=5 ; 2$ female/3 male; mean age of $58.6 \pm 8.1$ years; mean eGFR $\left.81.6 \pm 7.5 \mathrm{~mL} / \mathrm{min} / 1.73 \mathrm{~m}^{2}\right)$ or 'diseased' cortical tissue $(n=5 ; 3$ female $/ 2$ male; mean age of $54.8 \pm 3.3$ years; mean eGFR $81.2 \pm 8.0 \mathrm{~mL} / \mathrm{min} / 1.73 \mathrm{~m}^{2}$ ) based on the absence or presence of coexisting pathology in the nontumor kidney parenchyma (Table 1 and Figure 1A).

Amongst products of lipid peroxidation, 4-hydroxynonenal (4-HNE) is one of the most bioactive, and thus, is established as a biomarker of oxidative stress [10]. In line with the histological features of the kidney cortical tissue samples, immunohistochemical (IHC) staining demonstrated significantly 
elevated 4-HNE expression in 'diseased' specimens compared with 'normal' specimens (Figure 1B,C). Notably, we detected strong tubular positivity for 4-HNE in 'diseased' specimens, particularly localized to sites of pathological injury.

Table 1. Clinical and histological features of patients at the time of nephrectomy.

\begin{tabular}{ccccc}
\hline Patient & Age (Years)/Sex (M/F) & Primary Disease & eGFR & $\begin{array}{c}\text { Coexisting Pathology in } \\
\text { Nontumor Renal Parenchyma (\% } \\
\text { Diseased Cortical Area) }\end{array}$ \\
\hline & \multicolumn{2}{c}{ Normal PTEC $(n=5)$} & Nil \\
1 & $64 / \mathrm{F}$ & Clear cell RCC (Grade 2) & 72 & Nil \\
2 & $58 / \mathrm{M}$ & Clear cell RCC (Grade 2) & 77 & Nil \\
3 & $65 / \mathrm{F}$ & Clear cell RCC (Grade 2) & 81 & Nil \\
4 & $61 / \mathrm{M}$ & Clear cell RCC (Grade 2) & 88 & Nil \\
5 & $45 / \mathrm{M}$ & Clear cell RCC (Grade 2) & 90 & \\
& Diseased PTEC (n= 5) & & Glomerulosclerosis (5-10\%) \\
1 & $53 / \mathrm{M}$ & Chromophobe RCC & 75 & Glomerulosclerosis (5-10\%) \\
2 & $56 / \mathrm{F}$ & Chromophobe RCC & 90 & Arteriosclerosis (25-30\%) \\
3 & $52 / \mathrm{F}$ & Clear cell RCC (Grade 2) & 90 & Arteriosclerosis $(10-20 \%)$ \\
4 & $60 / \mathrm{M}$ & Clear cell RCC (Grade 4) & 75 & Tubular atrophy (10-20\%) \\
5 & $53 / \mathrm{F}$ & Chromophobe RCC & 76 & \\
\hline
\end{tabular}

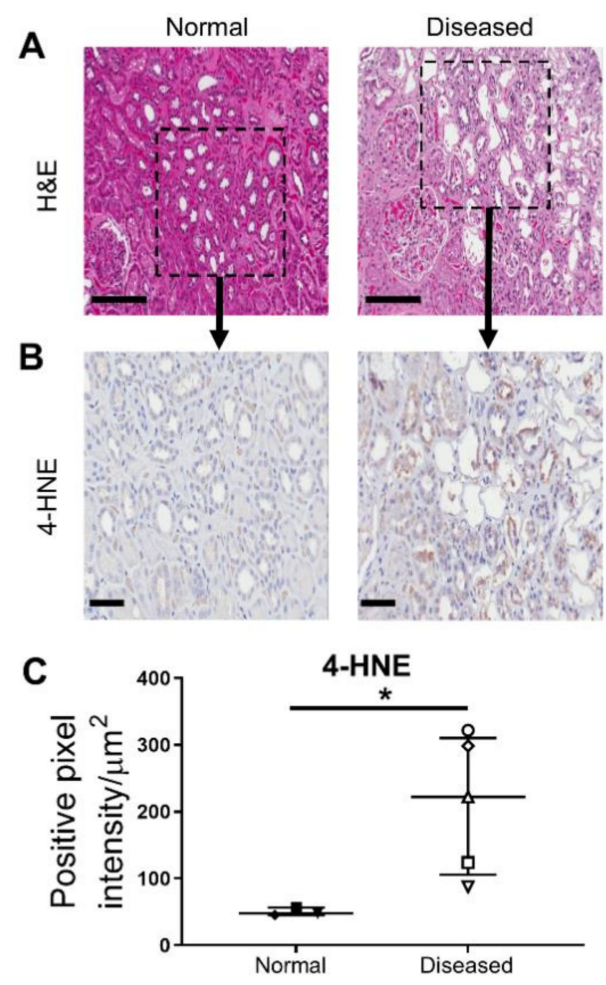

Figure 1. Significantly increased levels of oxidative stress in histologically 'diseased' cortical tissue. (A) Hematoxylin (purple) and eosin (pink) (H\&E) staining of tissue sections from histologically 'normal' (left panel) and 'diseased' cortical tissue (right panel) under light microscopy. Representative images from one of five 'normal' tissue samples and one of five 'diseased' tissue samples. Scale bars represent $200 \mu \mathrm{m}$. (B) IHC labelling of histologically 'normal' (left panel) and 'diseased' cortical tissue (right panel) probed for 4-hydroxynonenal (4-HNE). IHC staining images from representative areas indicated by the black squares in Figure $1 \mathrm{~A}$ are presented. Scale bars represent $60 \mu \mathrm{m}$. (C) Quantitative analysis (positive pixel intensity $/ \mu \mathrm{m}^{2}$ area) of 4-HNE staining in histologically 'normal' and 'diseased' cortical tissue. Symbols represent individual donor PTEC; $n=3$ 'normal' and $n=5$ 'diseased' tissue samples. Horizontal bars represent medians, with interquartile range also presented. ${ }^{*} p<0.05$, Mann-Whitney test. 


\subsection{Normal and Diseased PTEC Display Comparable Morphological and Molecular Profiles}

Human primary PTEC were subsequently isolated and cultured from these specimens. Initial experiments were performed to examine the morphology and molecular signature of PTEC isolated from cortical tissue with no histological indication of renal pathology (defined as normal PTEC) in comparison to those isolated from cortical tissue with histological evidence of renal pathology (diseased PTEC). Both normal and diseased PTEC displayed characteristic cobblestone morphology (Figure 2A). Expression levels of epithelial marker E-cadherin were similar between normal and diseased PTEC, with no or low expression of mesenchymal marker $\alpha$-smooth muscle actin ( $\alpha$-SMA) in both normal and diseased PTEC (Figure 2B,C). The expressions of nuclear factor erythroid 2-related factor 2 (Nrf2), a key regulator of the cellular antioxidant response [11], and $\gamma-\mathrm{H} 2 \mathrm{AX}$, a histone protein that contributes to chromatin-remodeling and responds to double-strand DNA breaks [12], were also comparable between the two PTEC groups (Figure 2B,C).

\section{A}

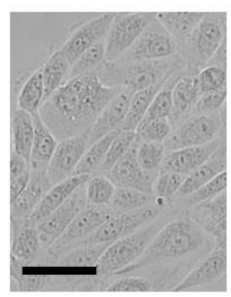

Normal PTEC
B

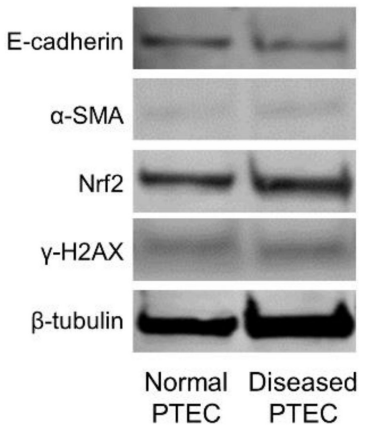

C

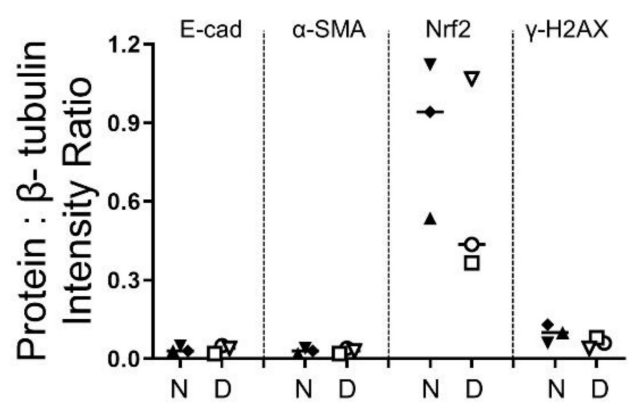

Figure 2. Human primary PTEC isolated and cultured from histologically normal and diseased cortical tissue display similar morphological and molecular profiles. (A) Light microscopy images of normal and diseased PTEC. Representative images from one of five normal PTEC and one of five diseased PTEC are shown. Scale bars represent $50 \mu \mathrm{m}$. (B) Western blot for E-cadherin, $\alpha$-SMA, Nrf2, and $\gamma-\mathrm{H} 2 \mathrm{AX}$ in whole cell extracts $(20 \mu \mathrm{g}$ total protein) from normal and diseased PTEC. Representative data from one of three normal PTEC and one of three diseased PTEC are presented. Full unedited gels available in the Supplementary File. (C) Relative expression of E-cadherin, $\alpha$-SMA, Nrf2, and $\gamma$-H2AX (protein intensity as a ratio of loading control $\beta$-tubulin) in normal (N) and diseased (D) PTEC. Symbols represent individual donor PTEC; $n=3$ donor PTEC per group.

\subsection{Normal and Diseased PTEC Phenotypically Respond to $\mathrm{H}_{2} \mathrm{O}_{2}$-Induced Oxidative Stress}

We next examined the phenotypic response of normal and diseased PTEC cultured in the absence (0 $\mathrm{mM}$ hydrogen peroxide; $\left.\mathrm{H}_{2} \mathrm{O}_{2}\right)$ and presence of low-level $\left(0.4 \mathrm{mM} \mathrm{H}_{2} \mathrm{O}_{2}\right)$ or high-level $(0.8 \mathrm{mM}$ $\mathrm{H}_{2} \mathrm{O}_{2}$ ) oxidative stress. Increased ROS production is a hallmark of oxidative stress, with superoxide the primary ROS species formed within mitochondria [13]. Baseline mitochondrial superoxide levels (\% MitoSOX ${ }^{+}$cells) were similar between normal and diseased PTEC under control $\left(0 \mathrm{mM} \mathrm{H}_{2} \mathrm{O}_{2}\right)$ conditions (Figure 3B). Both normal and diseased PTEC responded to $\mathrm{H}_{2} \mathrm{O}_{2}$-induced oxidative stress with an increase in mitochondrial superoxide levels (Figure 3A,B). For both PTEC groups, the fold change in $\mathrm{MitoSOX}^{+}$cells during high-level oxidative stress was significantly elevated compared with control $\left(0 \mathrm{mM} \mathrm{H}_{2} \mathrm{O}_{2}\right)$ conditions (Figure $\left.3 \mathrm{~A}, \mathrm{~B}\right)$.

Both normal and diseased PTEC retained expression of E-cadherin under conditions of low- and high-level oxidative stress (Figure 4A), with no significant change in E-cadherin levels observed under oxidative stress conditions compared with control conditions (Figure 4B). $\alpha$-SMA and Nrf2 levels in normal and diseased PTEC were minimally affected by $\mathrm{H}_{2} \mathrm{O}_{2}$-induced oxidative stress (Figure $4 \mathrm{~A}, \mathrm{C}, \mathrm{D}$ ). In contrast, DNA damage marker $\gamma-\mathrm{H} 2 \mathrm{AX}$ was significantly increased in both PTEC groups during high-level oxidative stress compared with control conditions (Figure 4A,E). These collective data confirm the capacity of both normal and diseased PTEC to respond to $\mathrm{H}_{2} \mathrm{O}_{2}$-induced oxidative stress. 


\subsection{Diseased PTEC Maintain Mitochondrial Function under Oxidative Stress Conditions}

We next examined the mitochondrial signatures of normal and diseased PTEC during oxidative stress using 5,5',6,6'-tetrachloro-1,1' ,3,3'-tetraethylbenzimi-dazolcarbocyanine iodide (JC-1) dye. This mitochondrial dye normally exists in solution as a monomer emitting a green fluorescence. However, in a reaction driven by MMP, JC-1 adopts a dimeric configuration emitting a red fluorescence [14,15]. Thus, JC-1 staining allows concurrent assessment of mitochondrial mass (green fluorescence) and MMP (red fluorescence). During high-level oxidative stress, both normal and diseased PTEC displayed significantly elevated mitochondrial mass (Figure 5A). Notably, whilst the MMP of normal PTEC was significantly attenuated during high-level oxidative stress, diseased PTEC maintained MMP levels under equivalent conditions (Figure 5B,C). These results indicate that diseased PTEC are capable of maintaining mitochondrial function, and thus, cellular health, during oxidative stress.

A

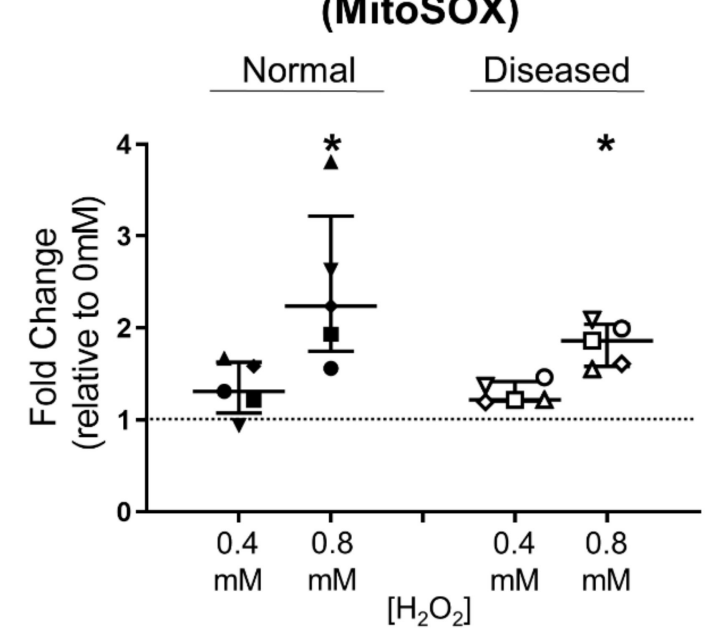

B

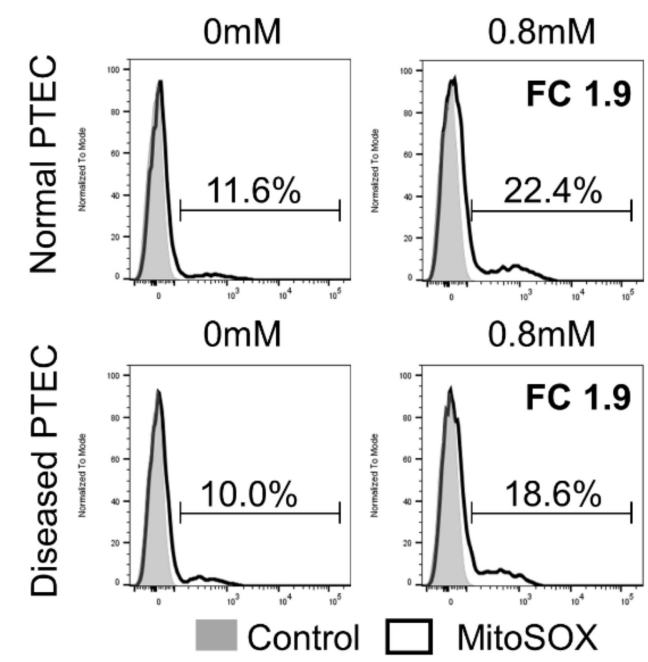

Figure 3. Significantly elevated mitochondrial superoxide levels in normal and diseased PTEC during high-level oxidative stress. (A) Fold changes (relative to $0 \mathrm{mM} \mathrm{H}_{2} \mathrm{O}_{2}$ cells) in mitochondrial superoxide levels (\% MitoSOX ${ }^{+}$cells) in normal and diseased PTEC cultured under low-level $\left(0.4 \mathrm{mM} \mathrm{H}_{2} \mathrm{O}_{2}\right)$ and high-level $\left(0.8 \mathrm{mM} \mathrm{H}_{2} \mathrm{O}_{2}\right)$ oxidative stress conditions. The dashed line represents a fold change of 1 . Symbols represent individual donor PTEC; $n=5$ donor PTEC per group. Horizontal bars represent medians, with interquartile range also presented. ${ }^{*} p<0.05$ vs $0 \mathrm{mM} \mathrm{H}_{2} \mathrm{O}_{2}$, Friedman test with Dunn's post-test. (B) Representative donor histograms of MitoSOX staining (black unfilled) in normal and diseased PTEC cultured in the absence $(0 \mathrm{mM})$ and presence $(0.8 \mathrm{mM})$ of $\mathrm{H}_{2} \mathrm{O}_{2}$ compared with unstained control (grey filled). Mitochondrial superoxide levels ( $\%$ MitoSOX ${ }^{+}$cells) are presented for each histogram, with fold change (FC) values relative to $0 \mathrm{mM} \mathrm{H}_{2} \mathrm{O}_{2}$ cells also shown. 
A

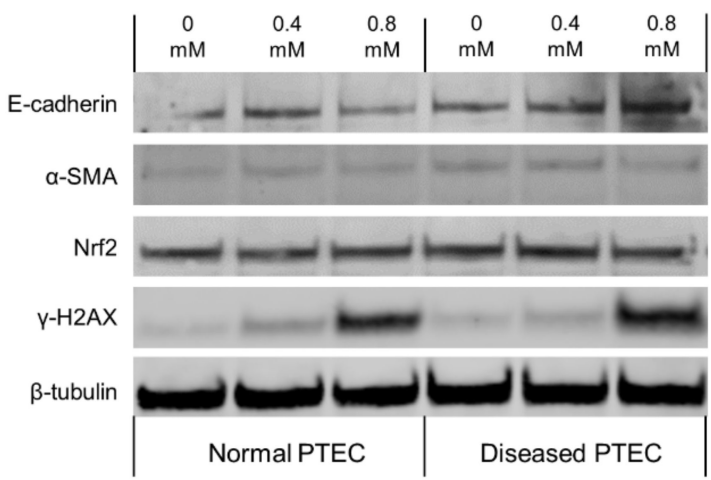

B

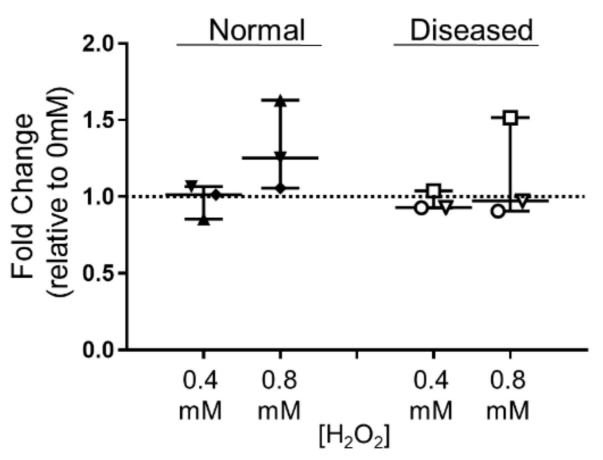

D

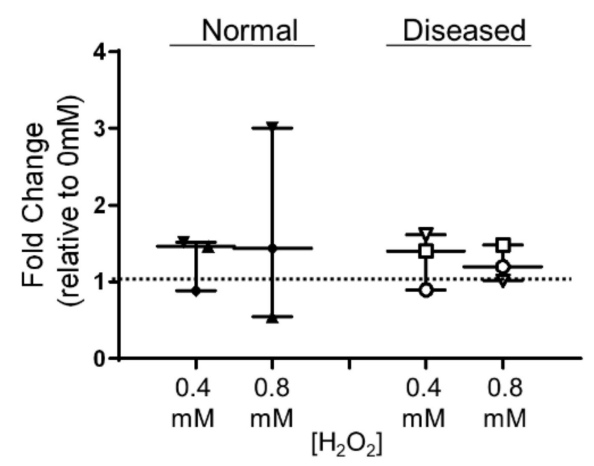

C

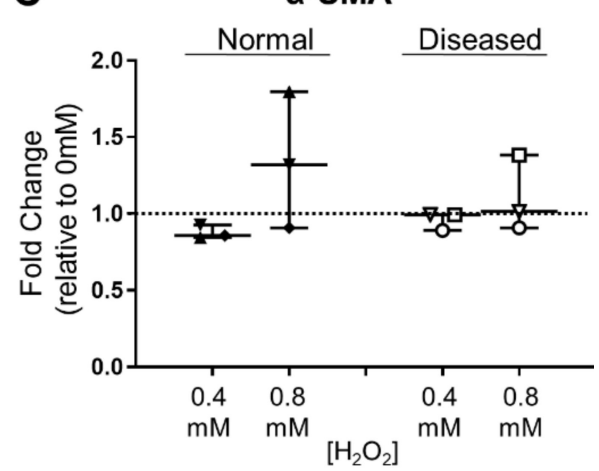

E

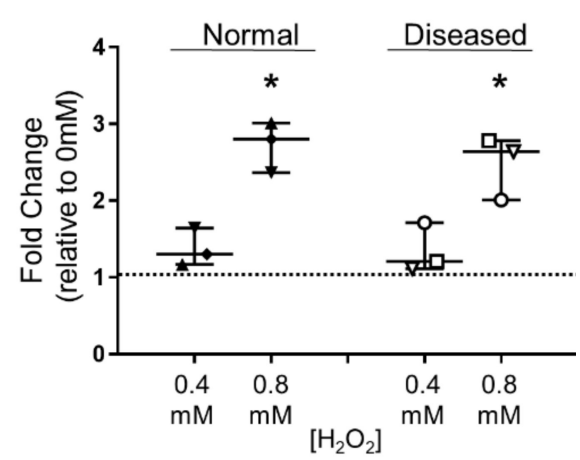

Figure 4. Significantly elevated DNA damage response in normal and diseased PTEC during high-level oxidative stress. (A) Western blot for E-cadherin, $\alpha$-SMA, Nrf2 and $\gamma$-H2AX in whole cell extracts (20 $\mu \mathrm{g}$ total protein) from normal and diseased PTEC cultured in the absence $\left(0 \mathrm{mM} \mathrm{H}_{2} \mathrm{O}_{2}\right)$ or presence of low-level $(0.4 \mathrm{mM})$ and high-level $(0.8 \mathrm{mM}) \mathrm{H}_{2} \mathrm{O}_{2}$. Representative data from one of three normal PTEC and one of three diseased PTEC are presented. Full unedited gels available in the Supplementary File. (B-E) Fold changes (relative to $0 \mathrm{mM} \mathrm{H}_{2} \mathrm{O}_{2}$ cells) in E-cadherin (B), $\alpha$-SMA (C), Nrf2 (D), and $\gamma$-H2AX (E) protein levels in normal and diseased PTEC cultured under low-level $\left(0.4 \mathrm{mM} \mathrm{H}_{2} \mathrm{O}_{2}\right)$ and high-level $\left(0.8 \mathrm{mM} \mathrm{H}_{2} \mathrm{O}_{2}\right)$ oxidative stress conditions. The dashed line represents a fold change of 1 . Symbols represent individual donor PTEC; $n=3$ donor PTEC per group. Horizontal bars represent medians, with interquartile range also presented. ${ }^{*} p<0.05$ vs $0 \mathrm{mM} \mathrm{H}_{2} \mathrm{O}_{2}$, Friedman test with Dunn's post-test. 
A

A Mitochondrial Mass (JC-1 Green)

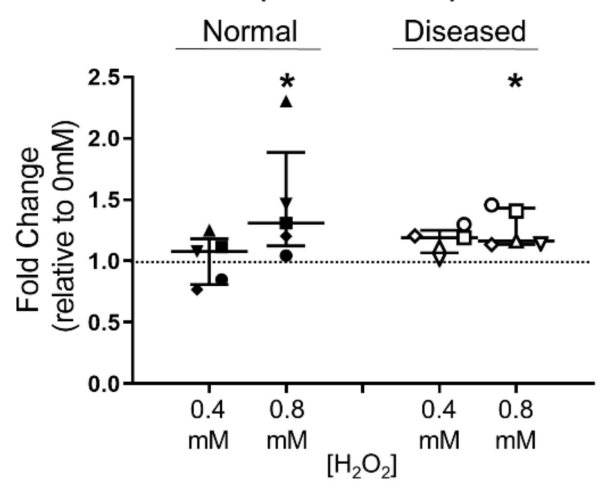

B
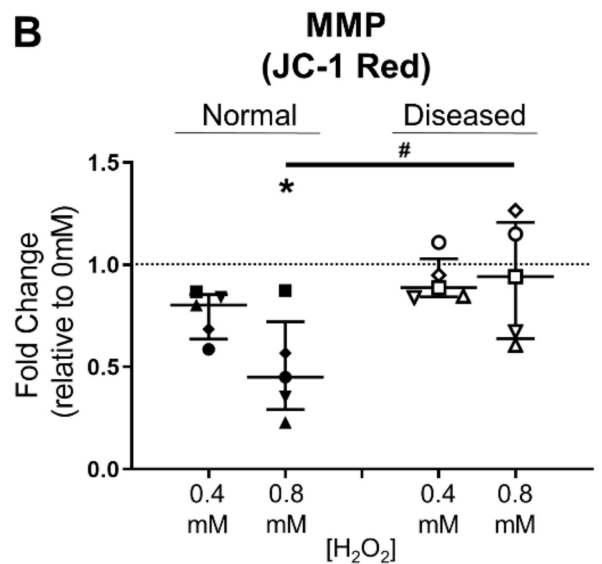

C

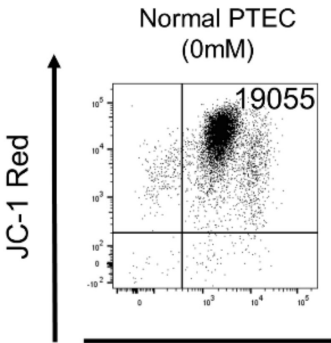

Normal PTEC $(0.8 \mathrm{mM})$

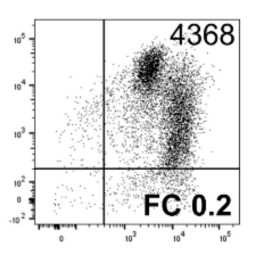

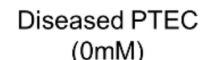
(OmM)

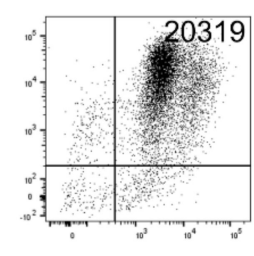

Diseased PTEC $(0.8 \mathrm{mM})$

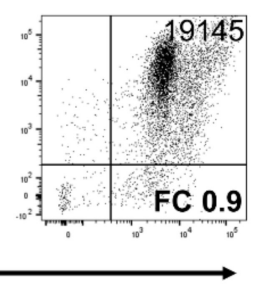

JC-1 Green

Figure 5. Diseased PTEC maintain mitochondrial function under high-level oxidative stress conditions. (A,B) Fold changes (relative to $0 \mathrm{mM} \mathrm{H}_{2} \mathrm{O}_{2}$ cells) in mitochondrial mass (JC-1 green fluorescence) (A) and mitochondrial membrane potential (MMP) (JC-1 red fluorescence) (B) in normal and diseased PTEC cultured under low-level $\left(0.4 \mathrm{mM} \mathrm{H}_{2} \mathrm{O}_{2}\right)$ and high-level $\left(0.8 \mathrm{mM} \mathrm{H}_{2} \mathrm{O}_{2}\right)$ oxidative stress conditions. The dashed line represents a fold change of 1 . Symbols represent individual donor PTEC; $n=5$ donor PTEC per group. Horizontal bars represent medians, with interquartile range also presented. ${ }^{*} p<0.05$ vs $0 \mathrm{mM} \mathrm{H}_{2} \mathrm{O}_{2}$, Friedman test with Dunn's post-test; \# $p<0.05$, Mann-Whitney test. (C) Representative donor JC-1 dot plots of normal and diseased PTEC cultured in the absence $(0 \mathrm{mM})$ and presence $(0.8 \mathrm{mM})$ of $\mathrm{H}_{2} \mathrm{O}_{2}$. JC- 1 red delta median fluorescence intensity (MFI) values (MFI test-MFI unstained control) are presented for each dot plot, with fold change (FC) values relative to $0 \mathrm{mM} \mathrm{H}_{2} \mathrm{O}_{2}$ cells also shown.

\subsection{Diseased PTEC Maintain Cell Viability under Oxidative Stress Conditions}

We next examined the functional impact (proliferation, viability) of these differential PTEC mitochondrial signatures. Both normal and diseased PTEC displayed significantly reduced cell proliferation under conditions of high-level oxidative stress compared with control conditions (Figure 6A).

PTEC death was also assessed in this in vitro model by Annexin-V/propidium iodide (PI) staining. We detected significantly increased levels of cell necrosis (\% Annexin- $\mathrm{V}^{+} \mathrm{PI}^{+}$cells) in normal PTEC during high-level oxidative stress compared with control conditions (Figure 6B,C). In contrast, diseased PTEC maintained cell viability under equivalent injurious conditions (Figure $6 \mathrm{~B}, \mathrm{C}$ ). Collectively, these data suggest that human PTEC from diseased cortical tissue display resistance to high-level oxidative stress, which, in turn, preserves cell viability. 
A

Proliferation

(MTT)

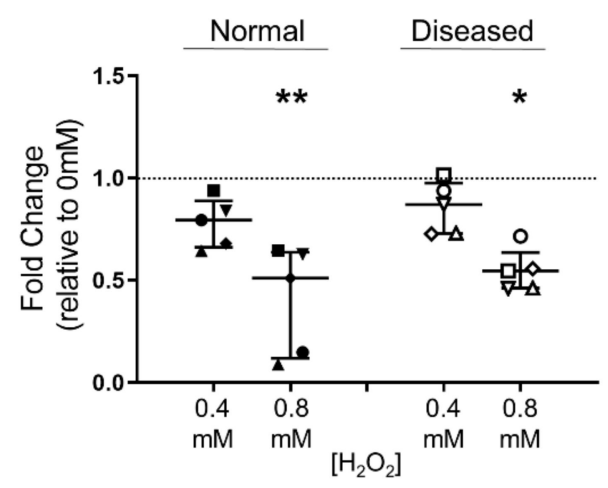

C

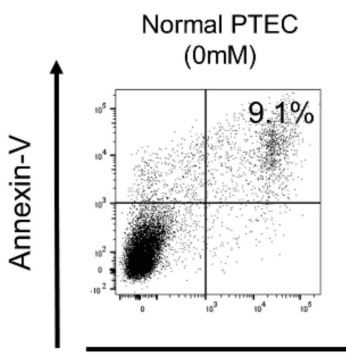

B

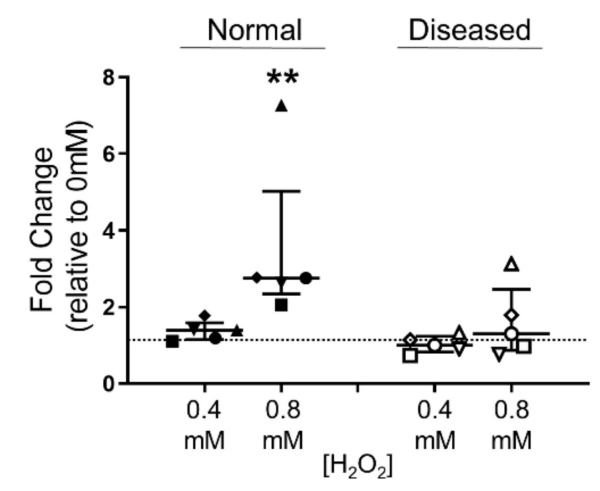

Normal PTEC

(0.8mM)

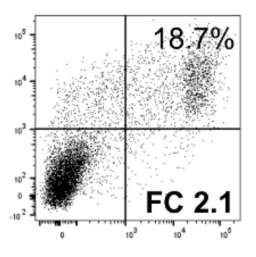

Propidium lodide
Diseased PTEC Diseased PTEC

(OmM)

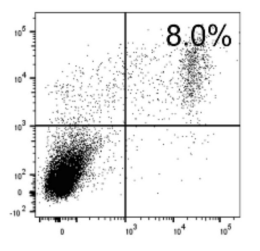

(0.8mM)

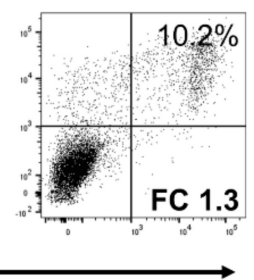

Figure 6. Diseased PTEC maintain cell viability under high-level oxidative stress conditions. (A,B) Fold changes (relative to $0 \mathrm{mM} \mathrm{H} \mathrm{O}_{2}$ cells) in cell proliferation (MTT assay) (A) and PTEC necrosis (\% Annexin- $\mathrm{V}^{+} \mathrm{PI}^{+}$cells) (B) in normal and diseased PTEC cultured under low-level $(0.4 \mathrm{mM}$ $\left.\mathrm{H}_{2} \mathrm{O}_{2}\right)$ and high-level $\left(0.8 \mathrm{mM} \mathrm{H}_{2} \mathrm{O}_{2}\right)$ oxidative stress conditions. The dashed line represents a fold change of 1 . Symbols represent individual donor PTEC; $n=5$ donor PTEC per group. Horizontal bars represent medians, with interquartile range also presented. ${ }^{*} p<0.05$, ${ }^{* *} p<0.01$ vs $0 \mathrm{mM} \mathrm{H}_{2} \mathrm{O}_{2}$, Friedman test with Dunn's post-test. (C) Representative donor Annexin-V/PI dot plots of normal and diseased PTEC cultured in the absence $(0 \mathrm{mM})$ and presence $(0.8 \mathrm{mM})$ of $\mathrm{H}_{2} \mathrm{O}_{2}$. The percentage of Annexin- $\mathrm{V}^{+} \mathrm{PI}^{+}$necrotic cells for each dot plot are presented, with fold change (FC) values relative to $0 \mathrm{mM} \mathrm{H}_{2} \mathrm{O}_{2}$ cells also shown.

\section{Discussion}

The response of PTEC to injurious stimuli, including oxidative stress, plays a central role in the progression of human kidney disease [16,17]. The research implicating PTEC in disease progression comes predominantly from animal models or in vitro studies using transformed cell lines. To date, translation of this work to human primary PTEC has been hampered by limited access to kidney cortical tissue samples. Furthermore, until now, the contribution of the underlying histopathology of the cortical tissue to the functional response of human primary PTEC has not been established. Here, we demonstrate for the first time that, in contrast to human primary PTEC from histologically 'normal' tissue, PTEC from histologically 'diseased' tissue are more resistant to high-level oxidative stress, with a preservation of mitochondrial function (retention of MMP) and cell viability.

The mitochondrial electron-transport chain is the major source of ROS (particularly the superoxide anion) during normal cellular metabolism [13]. However, the rate of mitochondrial ROS production is significantly increased under pathological conditions. This excessive generation of ROS leads to oxidative stress, ultimately inducing pathways of metabolic dysfunction and cell damage [7]. PTEC are particularly sensitive to oxidative stress due to their high metabolic demands required to reabsorb $80 \%$ of the filtrate (glucose, ions, etc.) that passes through the glomerulus [4]. We employed $\mathrm{H}_{2} \mathrm{O}_{2}$ to examine the intracellular PTEC response to oxidative stress in this present study, with $\mathrm{H}_{2} \mathrm{O}_{2}$ having been established as a common mediator of kidney disease, and also readily permeable to the plasma 
membrane [18]. Previous investigations have examined pathways of $\mathrm{H}_{2} \mathrm{O}_{2}$-induced oxidative stress in HK-2 cells, an immortalized human PTEC line [19-23]. Our present study is the first to demonstrate the induction of mitochondrial ROS (superoxide), and thus, oxidative stress in human primary PTEC in a model of $\mathrm{H}_{2} \mathrm{O}_{2}$-mediated injury. Notably, mitochondrial superoxide levels were significantly elevated in normal and diseased PTEC, confirming the capacity of both PTEC groups to respond to this injurious stimulus.

A significant consequence of oxidative stress is DNA damage. One of the earliest responses to DNA damage is the phosphorylation of the histone $\mathrm{H} 2 \mathrm{~A}$ variant $\mathrm{H} 2 \mathrm{AX}$ on a serine which is four residues from the $C$-terminus (residue 139) to form $\gamma$-H2AX [12]. As a consequence, $\gamma-\mathrm{H} 2 \mathrm{AX}$ formation has become a powerful and sensitive biomarker for the quantification of DNA damage. Here, for the first time, we use this biomarker to describe the DNA damage response of human primary PTEC to conditions of oxidative stress. In line with mitochondrial superoxide levels in our model of $\mathrm{H}_{2} \mathrm{O}_{2}$-mediated injury, we report significantly increased $\gamma-\mathrm{H} 2 \mathrm{AX}$ in both normal and diseased PTEC during high-level oxidative stress.

DNA damage as a consequence of excessive ROS generation can lead to epithelial-to-mesenchymal transition (EMT) [24]. EMT of tubular epithelial cells is defined by loss of epithelial cell characteristics (reduced E-cadherin) and acquisition of an extracellular matrix-producing myofibroblast phenotype (elevated expression of $\alpha$-SMA) [25-27]. EMT is a well-established mechanism in the development of tubulointerstitial fibrosis. $\mathrm{H}_{2} \mathrm{O}_{2}$-mediated oxidative stress has been previously shown to induce EMT in rat proximal tubular epithelial cells (NRK-52E) [28,29]. However, we did not identify the phenotypic switch characteristic of EMT induction (decreased E-cadherin/increased $\alpha$-SMA) in our human model of $\mathrm{H}_{2} \mathrm{O}_{2}$-mediated injury for either normal or diseased PTEC.

Despite the equivalent phenotypic profiles of normal and diseased PTEC during oxidative stress, the functional responses (mitochondrial function, viability) of these two PTEC groups were dissimilar. The functional response of normal PTEC to oxidative stress paralleled the reported pathogenesis of human kidney disease. It is established in human acute kidney injury (AKI) and chronic kidney disease (CKD) that the vicious cycle between oxidative stress and mitochondrial dysfunction critically contributes to tubular cell death, and thus, loss of kidney function [30,31]. In agreement with this concept, human primary PTEC from normal cortical tissue exhibited mitochondrial dysfunction (reduced MMP) and cell necrosis in response to $\mathrm{H}_{2} \mathrm{O}_{2}$-induced oxidative stress.

In contrast, PTEC from diseased cortical tissue were more resistant to high-level oxidative stress, with a maintenance of both mitochondrial function and cell viability. These data are reminiscent of senescent tubular epithelial cells in diseased kidneys [32,33]. Senescent cells undergo a program of permanent growth arrest and resistance to cell death in response to injurious stimuli, including oxidative stress [34]. Senescent cells accumulate and persist in injured organs as a consequence of maladaptive repair and are increasingly recognized as drivers of disease progression [32]. Notably, the accumulation of senescent tubular epithelial cells has been associated with histopathological changes (glomerulosclerosis, tubular atrophy, interstitial fibrosis) and loss of kidney function in human renal diseases [33]. Having been isolated from an analogous in vivo histopathological and oxidative stress-rich microenvironment (see Table 1 and Figure 1), we speculate that the diseased human primary PTEC in our study are more prone to entering a maladaptive program of cellular senescence upon further injury, as evidenced by their resistance to in vitro oxidative stress. This concept may have significant physiological relevance to the pathogenic mechanisms underlying human kidney diseases. We propose that the equivalent in vivo survival and accumulation of diseased PTEC in response to repeated oxidative stressors may be pivotal in preventing tubular regeneration, and thus, amplifying the loss in kidney function observed during AKI and CKD.

These novel results have significant implications for the therapeutic use of antioxidants in the treatment of human kidney diseases. Our findings suggest that the clinical efficacy of antioxidant compounds may be hindered by maladaptive PTEC in diseased microenvironments that are inherently resistant to oxidative stressors. We instead propose the therapeutic testing of senolytic agents that 
selectively induce death of senescent cells and, in turn, allow proliferation and regeneration of neighbouring tubular epithelial cells following kidney damage. For instance, the repurposing of senolytic drugs currently in use in clinical oncology (e.g. ABT-263; BCL-2, BCL-XL, and BCL-w inhibitor) may be of therapeutic benefit for targeting cellular senescence in the diseased kidney [35]. Candidate drugs such as these could be screened in the in vitro oxidative stress model presented in this study to assay their efficacy as senolytic agents on human primary PTEC.

Clinical translation of our novel findings will be dependent on the identification of the intracellular signaling pathways mediating this functional resistance in diseased PTEC. Indeed, in our in vitro model system, we examined the role of Nrf2, a critical transcription factor that controls the expression of cytoprotective proteins (e.g., heme oxygenase-1; HO-1) involved in the antioxidant response [36]. Previou1s studies have reported a protective role for Nrf2 in preventing tubular disease progression [37,38]. Despite evidence of preexisting oxidative stress in 'diseased' cortical tissue (Figure 1B), we detected comparable Nrf2 expression levels between normal and diseased PTEC under baseline conditions and in response to in vitro oxidative stress. Moreover, we were unable to detect HO-1 protein by Western blot under steady-state or oxidative stress conditions in either normal or diseased PTEC (data not shown). Further functional examination of Nrf2 and other stress-activated Nrf family members (i.e., Nrf1, Nrf3) in the cellular response of human primary PTEC to oxidative stress is now required.

Future work is also essential to examine the translatability of our findings to other non- $\mathrm{H}_{2} \mathrm{O}_{2}$ drivers of oxidative damage and mitochondrial dysfunction in human PTEC, including high-glucose, elevated albumin, inflammation, and hypoxia [8]. Particular emphasis should be placed on the relative response of normal versus diseased PTEC to nephrotoxic medicines that interfere with mitochondrial function and lead to oxidative stress, including antimicrobials (gentamicin, tetracycline), chemotherapeutic drugs (doxorubicin, cisplatin), and other medications (nonsteroidal anti-inflammatory drugs) [39].

Collectively, these results provide the first comprehensive characterization of primary PTEC isolated from normal and diseased human kidneys. A deeper mechanistic understanding of the distinct functional response of PTEC from diseased microenvironments to oxidative stress will enable the development of novel therapeutics capable of targeting the accumulation of these maladaptive cells in human kidney diseases.

\section{Materials and Methods}

\subsection{Kidney Cortical Tissue Specimens}

Kidney cortical tissue was obtained with informed patient consent from the macroscopically healthy portion of RCC nephrectomies, following approval by the Royal Brisbane and Women's Hospital Human Research Ethics Committee (Reference Number 2002/011; Approved 4 November 2016). Kidney tissue was immediately divided for: 1) fixation in formalin for IHC analysis; and 2) isolation and culture of human primary PTEC.

\subsection{IHC Staining}

Paraffin-embedded $4 \mu \mathrm{m}$ sections were deparaffinized and rehydrated. Endogenous peroxidase activity was blocked with $1 \% \mathrm{H}_{2} \mathrm{O}_{2}$ for $10 \mathrm{~min}$. Heat-induced antigen retrieval was performed in $0.01 \mathrm{M}$ Citrate Buffer ( $\mathrm{pH}$ 6.0) for $5 \mathrm{~min}$ at $125^{\circ} \mathrm{C}$. Antigen retrieval was followed by a protein block with $2 \%$ bovine serum albumin (BSA) for $30 \mathrm{~min}$ at room temperature. Sections were probed with anti-4-hydroxynonenal (4-HNE) (Goat polyclonal IgG; Abcam, Cambridge, MA, USA) at $4{ }^{\circ} \mathrm{C}$ overnight. Tissue sections were washed and a goat horseradish peroxidase (HRP) polymer system (Biocare Medical, Pacheco, CA, USA) was applied according to the manufacturer's instructions. Peroxidase activity was developed with ImmPACT DAB peroxidase (Vector Laboratories, Burlingame, CA, USA) for 5 min. Sections were lightly counterstained with hematoxylin and mounted using DPX Mounting Media. Quantitative analysis (positive pixel intensity $/ \mu \mathrm{m}^{2}$ area) was undertaken from three randomly selected 
areas for each tissue sample using ImageScope (v. 12.2.2.5015, Leica Biosystems, Mt Waverley, VIC, Australia).

\subsection{Isolation and Culture of Human Primary PTEC}

PTEC were isolated from kidney cortical tissue specimens following the method of Glynne and Evans [40] and cultured in Defined Medium (DM) as previously described [41]. All PTEC were used in experiments at passage 3 .

\subsection{Induction of Oxidative Stress in Human Primary PTEC}

PTEC were seeded (100,000 cells/well in DM) in 24-well flat-bottom plates to allow overnight adherence and then further cultured for 48 hours (unless otherwise specified) in fresh DM in the absence $\left(0 \mathrm{mM} \mathrm{H}_{2} \mathrm{O}_{2}\right)$ or presence of preoptimized low-level $(0.4 \mathrm{mM})$ and high-level $(0.8 \mathrm{mM}) \mathrm{H}_{2} \mathrm{O}_{2}$ to mimic oxidative stress injury. PTEC were harvested by trypsin treatment and analyzed for protein expression by Western blotting. PTEC oxidative stress, mitochondrial function and viability were assessed by flow cytometry, with cell acquisition performed on an LSR Fortessa (BD Biosciences, San Jose, CA, USA) and data analyzed with FlowJo software (TreeStar, Ashland, OR, USA).

\subsection{Western Blotting}

PTEC were lysed with Pierce ${ }^{\mathrm{TM}}$ RIPA lysis buffer (Pierce Protein Biology/Thermo Fisher Scientific, Waltham, MA, USA) containing protease inhibitor (Sigma-Aldrich, St Louis, MO, USA) and protein content determined using the BCA protein assay (Pierce Protein Biology/Thermo Fisher Scientific). Polyacrylamide gel electrophoresis (PAGE) was undertaken using standard reagents from Invitrogen (Eugene, OR, USA). Samples were denatured for $5 \mathrm{~min}$ at $95^{\circ} \mathrm{C}$, loaded onto Bolt ${ }^{\mathrm{TM}} 4-12 \%$ Bis-Tris Plus Gels, run at 200V for $26 \mathrm{~min}$ and transferred to a nitrocellulose membrane at 10V for $68 \mathrm{~min}$. Membranes were blocked for 1 hour at room temperature using Odyssey ${ }^{\circledR}$ blocking buffer (LI-COR, Lincoln, NE, USA) and subsequently probed with primary antibodies (Ab) overnight at $4{ }^{\circ} \mathrm{C}$, including E-cadherin (Rabbit monoclonal IgG; Clone 24E10; Cell Signaling Technology, Danvers, MA, USA), $\alpha$-SMA (Mouse monoclonal IgG2a; Clone 1A4; Sigma-Aldrich), Nrf2 (Rabbit polyclonal IgG; Abcam), $\gamma$-H2AX (Mouse monoclonal IgG; Clone 9F3; Abcam) and $\beta$-tubulin (Rabbit polyclonal IgG; Abcam). Proteins were visualized with IRDye $800 \mathrm{CW}$ goat antimouse (Millennium Science, Mulgrave, VIC, Australia) or IRDye 680LT goat antirabbit (Millennium Science) using the Odyssey CLX (LI-COR). Quantitative analysis of protein intensities relative to loading control $\beta$-tubulin was performed using Image Studio v. 2.0 software (LI-COR, Lincoln, NE, USA).

\subsection{Mitochondrial Superoxide Detection}

Mitochondrial superoxide levels were evaluated using MitoSOX ${ }^{\mathrm{TM}}$ Red (Invitrogen) [42]. Briefly, harvested PTEC were incubated with MitoSOX reagent $\left(1 \mu \mathrm{M}, 37^{\circ} \mathrm{C}, 30 \mathrm{~min}\right)$ with mitochondrial superoxide levels (expressed as MitoSOX ${ }^{+}$cells) determined by flow cytometry.

\subsection{Assessment of Mitochondrial Changes}

Mitochondrial assessments were performed using cationic dye JC-1 (Invitrogen) [14,43]. Briefly, PTEC were cultured for $24 \mathrm{~h}$ in the absence or presence of $\mathrm{H}_{2} \mathrm{O}_{2}$. PTEC were harvested and incubated with JC-1 (10 $\mu \mathrm{L}$ of $200 \mu \mathrm{M}$ stock, $\left.37^{\circ} \mathrm{C}, 30 \mathrm{~min}\right)$, with JC-1 green fluorescence emission $(\sim 529 \mathrm{~nm})$ and red fluorescence emission ( $\sim 590 \mathrm{~nm}$ ) detected by flow cytometry. JC-1 green fluorescence, representing the monomeric form of JC- 1 and corresponding to mitochondrial mass, was calculated as the delta median fluorescence intensity (delta MFI) (MFI test-MFI unstained control). JC-1 red fluorescence, corresponding to the J-aggregate form of JC- 1 and an indicator of MMP, was similarly expressed as the delta MFI. 


\subsection{Annexin V/PI Viability Assay}

The Annexin-V Apoptosis Detection kit I (BD Biosciences) was used to assess PTEC viability. Briefly, harvested PTEC were incubated with Annexin V FITC and PI in binding buffer for 15 min at room temperature. The percentage of Annexin $-\mathrm{V}^{+} \mathrm{PI}^{+}$necrotic cells was determined by flow cytometry.

\subsection{Cell Proliferation Measurements}

Cell proliferation was investigated using the colorimetric MTT (3-(4,5-dimethylthiazol2-yl)-2,5-diphenyltetrazolium bromide) Cell Proliferation Assay kit (Molecular Probes, Eugene, OR, USA). PTEC were seeded (20,000 cells/well in DM) in triplicate in 96-well flat-bottom plates to allow overnight adherence, and then further cultured for $48 \mathrm{~h}$ in fresh DM in the absence $\left(0 \mathrm{mM} \mathrm{H}_{2} \mathrm{O}_{2}\right)$ or presence of low-level $\left(0.4 \mathrm{mM} \mathrm{H}_{2} \mathrm{O}_{2}\right)$ and high-level $\left(0.8 \mathrm{mM} \mathrm{H}_{2} \mathrm{O}_{2}\right)$ oxidizing conditions. The MTT solution $(10 \mu \mathrm{L}$ of $12 \mathrm{mM}$ stock) was administered to PTEC, followed by a $2.5 \mathrm{~h}$ incubation at $37^{\circ} \mathrm{C}$. The MTT-containing medium was subsequently removed and dimethyl sulfoxide (DMSO) (Sigma-Aldrich) applied to the cells, followed by a $10 \mathrm{~min}$ incubation at $37^{\circ} \mathrm{C}$. Absorbance values at $540 \mathrm{~nm}$ were determined using a Powerwave X52 microplate reader (BioTek Instruments, Winooski, VT, USA).

\subsection{Statistics}

All data were normalized as a fold change relative to untreated control $\left(0 \mathrm{mM} \mathrm{H} \mathrm{H}_{2}\right)$ levels. All statistical tests were performed using Prism v. 7.0 analysis software (GraphPad Software, La Jolla, CA, USA). Comparisons between unpaired groups were performed using a Mann-Whitney test and multiple paired comparisons were performed using a Friedman test with Dunn's post-test. $P$ values $\leq 0.05$ were considered statistically significant.

Supplementary Materials: Supplementary Materials can be found at http://www.mdpi.com/1422-0067/21/2/560/s1.

Author Contributions: Each author has participated sufficiently in the work to take public responsibility for the content. M.A.K., J.U., W.H., H.H., G.G. and A.J.K. conceived and designed the study; M.A.K., X.W., K.T.K.G., P.N., A.G. and A.J.K. carried out experiments and analyzed the data; M.A.K., H.H., G.G. and A.J.K. drafted the paper. All authors revised and approved the final version of the manuscript.

Funding: The work was funded by Pathology Queensland and National Health and Medical Research Council (NHMRC) Project Grants (GNT1099222 and GNT1161319) and by the NHMRC Chronic Kidney Disease Centre of Research Excellence. M.A.K. was supported by a University of Queensland Research Training Program (RTP) Scholarship. K.T.K.G. was supported by an Australian Government RTP Scholarship.

Acknowledgments: The authors would like to thank the tissue donors and Queensland Health clinicians for providing histopathological assessments of nephrectomy specimens.

Conflicts of Interest: The authors declare no conflict of interest. The funders had no role in the design of the study; in the collection, analyses, or interpretation of data; in the writing of the manuscript, or in the decision to publish the results.

$\begin{array}{ll}\text { Abbreviations } \\ \mathrm{Ab} & \text { Antibody } \\ \mathrm{AKI} & \text { Acute kidney injury } \\ \mathrm{BSA} & \text { Bovine serum albumin } \\ \mathrm{CKD} & \text { Chronic kidney disease } \\ \mathrm{eGFR} & \text { Estimated glomerular filtration rate } \\ \mathrm{DM} & \text { Defined medium } \\ \mathrm{DMSO} & \text { Dimethyl sulfoxide } \\ \mathrm{EMT} & \text { Epithelial-to-mesenchymal transition } \\ \mathrm{H} \& \mathrm{E} & \text { Hematoxylin and eosin } \\ 4-\mathrm{HNE} & \text { 4-Hydroxynonenal } \\ \mathrm{HO}-1 & \text { Heme oxygenase-1 } \\ \mathrm{H}_{2} \mathrm{O}_{2} & \text { Hydrogen peroxide } \\ \mathrm{IHC} & \text { Immunohistochemical }\end{array}$




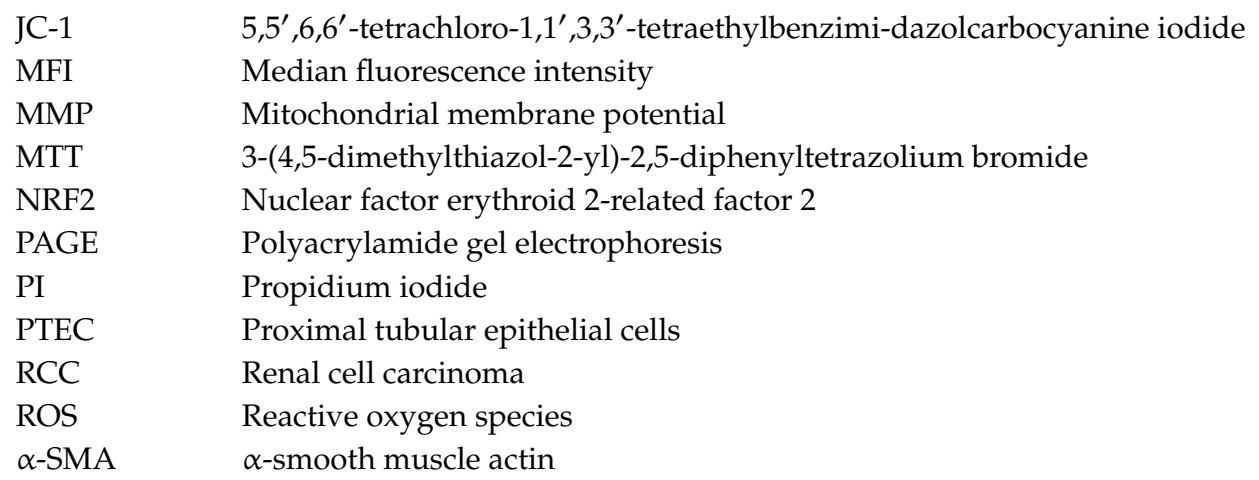

\section{References}

1. Gansevoort, R.T.; Correa-Rotter, R.; Hemmelgarn, B.R.; Jafar, T.H.; Heerspink, H.J.; Mann, J.F.; Matsushita, K.; Wen, C.P. Chronic kidney disease and cardiovascular risk: Epidemiology, mechanisms, and prevention. Lancet 2013, 382, 339-352. [CrossRef]

2. Xie, Y.; Bowe, B.; Mokdad, A.H.; Xian, H.; Yan, Y.; Li, T.; Maddukuri, G.; Tsai, C.Y.; Floyd, T.; Al-Aly, Z. Analysis of the Global Burden of Disease study highlights the global, regional, and national trends of chronic kidney disease epidemiology from 1990 to 2016. Kidney Int. 2018, 94, 567-581. [CrossRef] [PubMed]

3. Hommos, M.S.; Glassock, R.J.; Rule, A.D. Structural and Functional Changes in Human Kidneys with Healthy Aging. J. Am. Soc. Nephrol. 2017, 28, 2838-2844. [CrossRef] [PubMed]

4. Bhargava, P.; Schnellmann, R.G. Mitochondrial energetics in the kidney. Nat. Rev. Nephrol. 2017, 13, $629-646$. [CrossRef]

5. Daenen, K.; Andries, A.; Mekahli, D.; Van Schepdael, A.; Jouret, F.; Bammens, B. Oxidative stress in chronic kidney disease. Pediatr. Nephrol. 2019, 34, 975-991. [CrossRef]

6. Cross, C.E.; Halliwell, B.; Borish, E.T.; Pryor, W.A.; Ames, B.N.; Saul, R.L.; McCord, J.M.; Harman, D. Oxygen radicals and human disease. Ann. Intern. Med. 1987, 107, 526-545. [CrossRef]

7. Honda, T.; Hirakawa, Y.; Nangaku, M. The role of oxidative stress and hypoxia in renal disease. Kidney Res. Clin. Pract. 2019. [CrossRef]

8. Ratliff, B.B.; Abdulmahdi, W.; Pawar, R.; Wolin, M.S. Oxidant Mechanisms in Renal Injury and Disease. Antioxid. Redox Signal. 2016, 25, 119-146. [CrossRef]

9. Percy, C.J.; Power, D.; Gobe, G.C. Renal ageing: Changes in the cellular mechanism of energy metabolism and oxidant handling. Nephrology 2008, 13, 147-152. [CrossRef]

10. Breitzig, M.; Bhimineni, C.; Lockey, R.; Kolliputi, N. 4-Hydroxy-2-nonenal: A critical target in oxidative stress? Am. J. Physiol. Cell Physiol. 2016, 311, C537-C543. [CrossRef]

11. Ma, Q. Role of nrf2 in oxidative stress and toxicity. Annu. Rev. Pharmacol. Toxicol. 2013, 53, 401-426. [CrossRef] [PubMed]

12. Rogakou, E.P.; Pilch, D.R.; Orr, A.H.; Ivanova, V.S.; Bonner, W.M. DNA double-stranded breaks induce histone H2AX phosphorylation on serine 139. J. Biol. Chem. 1998, 273, 5858-5868. [CrossRef] [PubMed]

13. Murphy, M.P. How mitochondria produce reactive oxygen species. Biochem. J. 2009, 417, 1-13. [CrossRef] [PubMed]

14. Smiley, S.T.; Reers, M.; Mottola-Hartshorn, C.; Lin, M.; Chen, A.; Smith, T.W.; Steele, G.D., Jr.; Chen, L.B. Intracellular heterogeneity in mitochondrial membrane potentials revealed by a J-aggregate-forming lipophilic cation JC-1. Proc. Natl. Acad. Sci. USA 1991, 88, 3671-3675. [CrossRef]

15. Reers, M.; Smith, T.W.; Chen, L.B. J-aggregate formation of a carbocyanine as a quantitative fluorescent indicator of membrane potential. Biochemistry 1991, 30, 4480-4486. [CrossRef]

16. Sharfuddin, A.A.; Molitoris, B.A. Pathophysiology of ischemic acute kidney injury. Nat. Rev. Nephrol. 2011, 7, 189-200. [CrossRef]

17. Qi, R.; Yang, C. Renal tubular epithelial cells: The neglected mediator of tubulointerstitial fibrosis after injury. Cell Death Dis. 2018, 9, 1126. [CrossRef] 
18. Cui, X.L.; Ding, Y.; Alexander, L.D.; Bao, C.; Al-Khalili, O.K.; Simonson, M.; Eaton, D.C.; Douglas, J.G. Oxidative signaling in renal epithelium: Critical role of cytosolic phospholipase A2 and p38(SAPK). Free Radic. Biol. Med. 2006, 41, 213-221. [CrossRef]

19. Small, D.M.; Bennett, N.C.; Roy, S.; Gabrielli, B.G.; Johnson, D.W.; Gobe, G.C. Oxidative stress and cell senescence combine to cause maximal renal tubular epithelial cell dysfunction and loss in an in vitro model of kidney disease. Nephron Exp. Nephrol. 2012, 122, 123-130. [CrossRef]

20. Hou, X.; Xiao, H.; Zhang, Y.; Zeng, X.; Huang, M.; Chen, X.; Birnbaumer, L.; Liao, Y. Transient receptor potential channel 6 knockdown prevents apoptosis of renal tubular epithelial cells upon oxidative stress via autophagy activation. Cell Death Dis. 2018, 9, 1015. [CrossRef]

21. Lin, M.; Li, L.; Zhang, Y.; Zheng, L.; Xu, M.; Rong, R.; Zhu, T. Baicalin ameliorates H2O2 induced cytotoxicity in HK-2 cells through the inhibition of ER stress and the activation of Nrf2 signaling. Int. J. Mol. Sci. 2014, 15, 12507-12522. [CrossRef] [PubMed]

22. Andreucci, M.; Fuiano, G.; Presta, P.; Lucisano, G.; Leone, F.; Fuiano, L.; Bisesti, V.; Esposito, P.; Russo, D.; Memoli, B.; et al. Downregulation of cell survival signalling pathways and increased cell damage in hydrogen peroxide-treated human renal proximal tubular cells by alpha-erythropoietin. Cell Prolif. 2009, 42, 554-561. [CrossRef] [PubMed]

23. Lee, Y.M.; Shin, J.W.; Lee, E.H.; Moon, Y.; Seo, Y.J.; Kim, J.Y.; Kim, J.U. Protective effects of propofol against hydrogen peroxide-induced oxidative stress in human kidney proximal tubular cells. Korean J. Anesthesiol. 2012, 63, 441-446. [CrossRef] [PubMed]

24. Zhang, S.; Tan, X.; Chen, Y.; Zhang, X. Postconditioning protects renal fibrosis by attenuating oxidative stress-induced mitochondrial injury. Nephrol. Dial. Transplant. 2017, 32, 1628-1636. [CrossRef]

25. Ng, Y.Y.; Huang, T.P.; Yang, W.C.; Chen, Z.P.; Yang, A.H.; Mu, W.; Nikolic-Paterson, D.J.; Atkins, R.C.; Lan, H.Y. Tubular epithelial-myofibroblast transdifferentiation in progressive tubulointerstitial fibrosis in 5/6 nephrectomized rats. Kidney Int. 1998, 54, 864-876. [CrossRef]

26. Yang, J.; Liu, Y. Dissection of key events in tubular epithelial to myofibroblast transition and its implications in renal interstitial fibrosis. Am. J. Pathol. 2001, 159, 1465-1475. [CrossRef]

27. Iwano, M.; Plieth, D.; Danoff, T.M.; Xue, C.; Okada, H.; Neilson, E.G. Evidence that fibroblasts derive from epithelium during tissue fibrosis. J. Clin. Investig. 2002, 110, 341-350. [CrossRef]

28. Rhyu, D.Y.; Yang, Y.; Ha, H.; Lee, G.T.; Song, J.S.; Uh, S.T.; Lee, H.B. Role of reactive oxygen species in TGF-beta1-induced mitogen-activated protein kinase activation and epithelial-mesenchymal transition in renal tubular epithelial cells. J. Am. Soc. Nephrol. 2005, 16, 667-675. [CrossRef]

29. Wang, Y.; Pang, L.; Zhang, Y.; Lin, J.; Zhou, H. Fenofibrate Improved Interstitial Fibrosis of Renal Allograft through Inhibited Epithelial-Mesenchymal Transition Induced by Oxidative Stress. Oxid. Med. Cell. Longev. 2019, 2019. [CrossRef]

30. Ishimoto, Y.; Inagi, R. Mitochondria: A therapeutic target in acute kidney injury. Nephrol. Dial. Transplant. 2016, 31, 1062-1069. [CrossRef]

31. Che, R.; Yuan, Y.; Huang, S.; Zhang, A. Mitochondrial dysfunction in the pathophysiology of renal diseases. Am. J. Physiol.-Ren. Physiol. 2014, 306, F367-F378. [CrossRef] [PubMed]

32. Docherty, M.H.; O'Sullivan, E.D.; Bonventre, J.V.; Ferenbach, D.A. Cellular Senescence in the Kidney. J. Am. Soc. Nephrol. 2019, 30, 726-736. [CrossRef] [PubMed]

33. Valentijn, F.A.; Falke, L.L.; Nguyen, T.Q.; Goldschmeding, R. Cellular senescence in the aging and diseased kidney. J. Cell Commun. Signal. 2018, 12, 69-82. [CrossRef] [PubMed]

34. Colavitti, R.; Finkel, T. Reactive oxygen species as mediators of cellular senescence. IUBMB Life 2005, 57, 277-281. [CrossRef] [PubMed]

35. Knoppert, S.N.; Valentijn, F.A.; Nguyen, T.Q.; Goldschmeding, R.; Falke, L.L. Cellular Senescence and the Kidney: Potential Therapeutic Targets and Tools. Front. Pharmacol. 2019, 10, 770. [CrossRef]

36. Nguyen, T.; Nioi, P.; Pickett, C.B. The Nrf2-antioxidant response element signaling pathway and its activation by oxidative stress. J. Biol. Chem. 2009, 284, 13291-13295. [CrossRef]

37. Ruiz, S.; Pergola, P.E.; Zager, R.A.; Vaziri, N.D. Targeting the transcription factor Nrf2 to ameliorate oxidative stress and inflammation in chronic kidney disease. Kidney Int. 2013, 83, 1029-1041. [CrossRef]

38. Nezu, M.; Souma, T.; Yu, L.; Suzuki, T.; Saigusa, D.; Ito, S.; Suzuki, N.; Yamamoto, M. Transcription factor Nrf2 hyperactivation in early-phase renal ischemia-reperfusion injury prevents tubular damage progression. Kidney Int. 2017, 91, 387-401. [CrossRef] 
39. Eirin, A.; Lerman, A.; Lerman, L.O. The Emerging Role of Mitochondrial Targeting in Kidney Disease. Handb. Exp. Pharmacol. 2017, 240, 229-250. [CrossRef]

40. Glynne, P.A.; Evans, T.J. Inflammatory cytokines induce apoptotic and necrotic cell shedding from human proximal tubular epithelial cell monolayers. Kidney Int. 1999, 55, 2573-2597. [CrossRef]

41. Kassianos, A.J.; Sampangi, S.; Wang, X.; Roper, K.E.; Beagley, K.; Healy, H.; Wilkinson, R. Human proximal tubule epithelial cells modulate autologous dendritic cell function. Nephrol. Dial. Transplant. 2013, 28, 303-312. [CrossRef] [PubMed]

42. Mukhopadhyay, P.; Rajesh, M.; Yoshihiro, K.; Hasko, G.; Pacher, P. Simple quantitative detection of mitochondrial superoxide production in live cells. Biochem. Biophys. Res. Commun. 2007, 358, 203-208. [CrossRef] [PubMed]

43. Mancini, M.; Anderson, B.O.; Caldwell, E.; Sedghinasab, M.; Paty, P.B.; Hockenbery, D.M. Mitochondrial proliferation and paradoxical membrane depolarization during terminal differentiation and apoptosis in a human colon carcinoma cell line. J. Cell Biol. 1997, 138, 449-469. [CrossRef] [PubMed]

(C) 2020 by the authors. Licensee MDPI, Basel, Switzerland. This article is an open access article distributed under the terms and conditions of the Creative Commons Attribution (CC BY) license (http://creativecommons.org/licenses/by/4.0/). 